\title{
Food and Feeding, Length-Weight and Condition Factor of the Catfish Synodontis membranaceus (EtieneGeoffroy Saint Hilaire, 1809) (Osteichthyes: Mochokidae) from Lower Benue River, Makurdi, Nigeria
}

\author{
Atile John I., Shima Judith N., Akombo Pauline M. \\ Department of Biological Sciences, Benue State University, Makurdi, Nigeria \\ Email address: \\ johnatile@gmail.com (Atile J. I.), judith14real@yahoo.com (Shima J. N.), mbakaanpauline@yahoo.com (Akombo P. M.) \\ To cite this article: \\ Atile John I., Shima Judith N., Akombo Pauline M. Food and Feeding, Length-Weight and Condition Factor of the Catfish Synodontis \\ membranaceus (EtieneGeoffroy Saint Hilaire, 1809) (Osteichthyes: Mochokidae) from Lower Benue River, Makurdi, Nigeria. Agriculture, \\ Forestry and Fisheries. Vol. 5, No. 4, 2016, pp. 87-96. doi: 10.11648/j.aff.20160504.11
}

Received: March 7, 2016; Accepted: March 25, 2016; Published: July 13, 2016

\begin{abstract}
Food and Feeding, length-weight and condition factor of the Catfish Synodontis membranaceus from the Lower Benue River at Makurdi, Nigeria, was studied for 12- month period from July, 2013 to June, 2014. A total number of 202 specimens, comprising of 101 males and 101 females at a ratio of 1:1 were studied. The Length-Weight Relationship (LWR), correlation coefficient $(r)$ for all males and females were highly positive and significant at $(\mathrm{P}<0.01)$, with $\mathrm{r}$ values of 0.972 for males, 0.899 for females and 0.952 for combined sexes. The mean b values (regression coefficient) were 2.68 in all the males, 2.46 in all the females and 2.63 in combined sexes indicating that all the sexes had negative allometric growth pattern. The mean condition factor $(\mathrm{K})$ for both sexes ranged from 2.21-3.35. The lowest $\mathrm{K}$ range (1.81) for males was observed in July, 2013 while the highest K range (3.05) was observed in April, 2014. Similarly, the lowest K range (1.83) for females was observed in August, 2013 with the highest K range of 3.14 in the same month. Out of the 202 stomachs examined, only 9 males $(8.91 \%)$ and 5 females $(4.95 \%)$ had empty stomachs, about $91.09 \%$ of males and $95.0 \%$ of females had varied quantities of food items in their stomachs. Synodontis membranaceus in the Lower Benue River was found to be omnivorous, feeding on food items, which included artificial meal, plant remains, variety of algae, insect parts and larvae, bivalves (Molluscs), crustaceans, protozoa, worms, detritus, sand particles and mud and many unidentified quantities of food items.
\end{abstract}

Keywords: Synodontis membranaceus, Food, Feeding, Lower Benue River

\section{Introduction}

Fish, as all animals, require adequate nutrition in order to grow and survive. Some fish feed on plant materials; others feed only on animals, whereas a third and larger group derive their proteins, carbohydrates and fats, as well as vitamins and minerals necessary for their growth and maintenance, from both plant and animal sources (Lagler, et al., 1977). According to Akange (2011), no species of fish is associated with a particular kind of food item. Feeding of fish therefore, depends on the abundance and availability of potential food items (Lagler et al., 1977). S. membranaceus has been found to be a typical example of fish without strict feeding habit and it is regarded as omnivore, because of its ability to use any food material available in its habitat (Owolabi, 2008). The feeding habits of fish as well as its feeding habits influence its growth, behaviour and other ecological characteristics (Ogbe et al., 2008).

The study of food and feeding of fish based on stomach content analysis is widely used in fish biology and ecology to indicate the position of a species within a food web and to provide information on the contribution of different prey items to the diet (Bagenal, 1978). Food items consumed by fish are ascertained by carefully observing their stomach content especially after feeding. Data on stomach composition is vital in providing straight forward models of stomach content dynamics and formulating management strategy options in multi-species fishery (Adeyemi et al., 2009).

The study of the food and feeding of freshwater fish 
species is a subject of continuous research because it constitutes the basis for the development of a successful fisheries management programme on fish culture (Oransaye and Nakpochia, 2005). Berg (1979) reported that the analysis of stomach content of fish could provide information about the niche of a particular fish in its ecosystem and this has become a standard practice in fish ecology works.

Extensive research has been carried out on the Lower Benue River which includes that of Ogbe and Fagade (2002), Ogbe et al., (2008), Solomon et al., (2009), Akombo et al., (2011) on Hydrocynus forskalii, Alestes nurse and Synodontis respectively. However, So far, no extensive work has been done on the food and feeding habit of S. membranaceus from Lower Benue River, Nigeria. Hence there is a need to investigate food and feeding behavior of this fish in order to know more of its biological and physiological aspects to sustain fish farming of Synodontis membranaceus, through aquaculture industry which will provide biological and statistical information on the Lower Benue River Fisheries and as well as provide information on the formulation of fish diet relevant for aquaculture.

\section{Materials and Methods}

\subsection{Description of the Study Area}

The study was carried out in the Lower Benue River, Makurdi. The Lower Benue River as described by Reid and Sydenhan (1979) is the portion of the Benue River contained within the Benue State of Nigeria. River Benue originates mainly in the Adamawa Mountains of Cameroun, some 500 $\mathrm{km}$ beyond the Nigerian frontier, and flows west across EastCentral

Nigeria (Nedeco, 1959). It is the largest tributary of the Niger which joins at Lokoja. The River has extensive alluvial plain (uncommon in African Rivers) stretching for many kilometers, covering a distance of approximately $187 \mathrm{~km}$. This extensive flood plain forms breeding grounds for many fish species (Beadle, 1974). The highest water levels are in August to September and the lowest are in March to April (Akombo et al., 2011). (Be brief and concisein describing the study area)

\subsection{Sampling Method}

The fresh specimens of Synodontis membranaceus were purchased from the fishermen at Wurukum and Wadata markets with which the biggest fish are located in Makurdi. Bi-monthly purchasing of the specimens from the landing sites was taken for twelve months-period from July, 2013 to June, 2014. The fish samples purchased were then transported to the laboratory in plastic containers containing ice blocks to keep the fish fresh.

\subsection{Morphometric Measurements}

The morphometric parameters measured were length (SL) and body weights (BW). SLs of the specimens were determined by using a measuring board as described according to Akombo et al., (2011). The BW were monitored using a digital electronic weighing balance, (ADAM AFP 4100L). The specimens were weighed to the nearest $0.1 \mathrm{~g}$.

\subsection{Determination of Sex}

The sex of $S$. membranaceus was determined after dissecting the fish. Mid-ventral incision on the abdomen of the fish was performed from the anal opening to the end of opercula region to expose the internal organs. In the young males, testes are described as thin, thread -like with very small projections, whitish in colour and extend to about onethird of the abdominal cavity. In adult males, they are creamy in colour with very conspicuous granules. The young females have thin, pink to white tubular structures occupying about one-fifth of the portion of the body cavity. In adult females, eggs are readily discernable in the ovaries which increase in size and fill up most of the abdominal cavity (Bagenal, 1978; Halim and Guma'a, 1989). Sex ratio was calculated using the formula:

$$
\text { Sex ratio }=\frac{\text { Number of males }}{\text { Number of females }}
$$

\subsection{Fulton's Condition Factor (K)}

The condition factor $(\mathrm{K})$ for each specimen was computed from

$$
\mathrm{K}=100 / \mathrm{L}^{3}
$$

Where, $\mathrm{W}$ is the observed body weight for each specimen, $\mathrm{L}$ is the observed standard length for each specimen and $\mathrm{K}$ is the condition factor.

\subsection{The Length-Weight Relationship}

Length-Weight relationship in $S$. membranaceus was represented by:

$$
\mathrm{W}=\mathrm{aL}^{\mathrm{b}}
$$

Where $\mathrm{b}$ is an exponent usually between 2 and 4 according to Bagenal (1978). W is the observed total body weight, a is the intercept on the length axis and $\mathrm{L}$ is the observed standard length. The logarithmic transformation of equation 2 gives a straight line relationship

$$
\log W=\log a+b \log L
$$

Log weight is plotted against log length, the regression coefficient is $b$, and $\log a$ is the intercept of the line on the Yaxis.

\subsection{Determination of Food Items}

After dissecting the fish, the stomachs were removed and weighed using a digital electronic weighing balance. The stomachs were weighed to the nearest $0.1 \mathrm{~g}$ and preserved in $4 \%$ formalin for subsequent examination.

Each stomach sample was slit open and emptied in a clean petridish and some food items were identified 
macroscopically. Three slides preparations for each stomach content was made and examined under a light Olympus Camera microscope model-CX31RTSF using X4, X10 and $\mathrm{X} 40$ objectives lens to identify and take photographs of some microscopic food items. The stomach contents of the specimens were analysed by:

\section{$i$. Frequency of occurrence method}

Frequency of occurrence (FO) of food items were calculated using the formula:

$$
\text { FO }=\frac{\text { Number of stomachs with a food item }}{\text { Total number of non }- \text { empty stomachs }} \times \frac{100}{1}
$$

The values obtained were called percentage of occurrence of food items ( $\%$ OFI).

\section{ii. Numerical method}

Different types of items in the stomach content of the fish species were calculated under numerical method (NM) using the formula:

$$
\mathrm{NM}=\frac{\text { Food item }}{\text { Total number of different food items }} \times \frac{100}{1}
$$

The values obtained were called percentage composition of food items (\% CFI) by number (Bagenal, 1978).

\section{iii.Point method}

One hundred points were awarded to all the contents of a stomach and each food item was allotted with a number of points based on the macroscopic and microscopic judgments. All the points gained by a particular food item were expressed as a percentage of the total points scored by food items in the stomach samples thus point method (PM) was calculated using the formula:

$$
\mathrm{PM}=\frac{\text { Total points scored by a food item }}{\text { Total number of points of all food items }} \times \frac{100}{1}
$$

The values obtained were called percentage composition of food items by point method.

\subsection{Determination of Stomach Vacuity Index}

The stomachs were examinedmacroscopically and microscopically using three different slides preparations after they were slit opened to determine their emptiness or nonemptiness. Stomach vacuity index (SVI) was calculated using the formula:

$$
\text { SVI }=\frac{\text { Number of empty stomachs } X 100}{\text { Total number of stomachs }} \times \frac{100}{1}
$$

The values obtained were called percentage of stomach vacuity index (\% SVI).

\section{Results}

On the whole, male and female species of $S$. membranaceus from Lower Benue River from July, 2013 to June, 2014 had 50\% abundance each. However, males were slightly more than the females in some months, and vice versa. The species were not available during themonths of February and March, 2014 (Table 2). The highest standard length in males $(25.00 \mathrm{~cm})$ was observed in July, 2013 with a highest weight of $398.82 \mathrm{~g}$ in the same month. On the other hand, the highest standard length in females $(26.10 \mathrm{~cm})$ was observed in September, 2013 with a highest weight of 405.66g (Table 1). The relative condition factors of the species were observed to be higher in wet seasons compared withthe dry seasons. In males, the lowest $\mathrm{K}$ was observed in July, 2013 and January, 2014, while the highest $\mathrm{K}$ was observed in April, 2014. In females, the lowest and highest $\mathrm{K}$ was observed in August, 2014 (Figure 1). The logarithmic transformed length-weight relationship of male, female and combined sexes of $S$. membranaceus correlated positively and the correlation coefficients (r) were significant (Figure 2, 3 and 4). The stomach content analysis of S. membranaceus using the Frequency of Occurrence Method, the Point Method and the Numerical Method revealed that diatoms, surface water algae and insect parts were dominants food items consumed by the fish (Table 4, 5 and 6). On the other hand plant remains detritus, sand particles/mud and crustacean parts were the dominant food items using Frequency of Occurrence and Point Methods. The females' species of $S$. membranaceus had a higher stomach vacuity index than the males' species in July, 2013 and in September, 2013. A zero per cent stomach vacuity index was observed in females in August, 2013 while a higher stomach vacuity indices of both males and females was zero per cent in

\begin{tabular}{|c|c|c|c|c|}
\hline Month & Sex & No. & WT (g) & SL (cm) \\
\hline \multirow[t]{2}{*}{ July, 2013} & $\mathrm{M}$ & 27 & $52.42-398.82$ & $12.30-25.00$ \\
\hline & $\mathrm{F}$ & 17 & $69.61-379.89$ & $14.43-24.90$ \\
\hline \multirow[t]{2}{*}{ August, 2013} & M & 22 & $42.30-289.32$ & $11.60-23.20$ \\
\hline & $\mathrm{F}$ & 25 & $63.08-309.46$ & $13.70-24.50$ \\
\hline \multirow[t]{2}{*}{ September, 2013} & M & 12 & $107.50-191.52$ & $13.60-20.00$ \\
\hline & $\mathrm{F}$ & 15 & $77.25-405.66$ & $14.90-26.10$ \\
\hline October, 2013 & M & 1 & $0.00-118.42$ & $0.00-16.40$ \\
\hline \multirow[t]{2}{*}{ November, 2013} & M & 2 & $8.67-9.78$ & $7.20-7.60$ \\
\hline & $\mathrm{F}$ & 2 & $14.92-111.36$ & $8.80-17.10$ \\
\hline \multirow[t]{2}{*}{ December, 2013} & M & 1 & $0.00-8.67$ & $0.00-7.10$ \\
\hline & $\mathrm{F}$ & - & & - \\
\hline
\end{tabular}
October, 2013, November, 2013, December, 2013, January, 2014, April, 2014, May, 2014 and June, 2014 (Table 7).

Table 1. Sex distribution and standard length (SL) range of S. membranaceus in the Lower Benue River from July, 2013 to June, 2014. 


\begin{tabular}{lllll}
\hline Month & Sex & No. & WT (g) \\
\hline January, 2014 & M & 1 & $0.00-6.85$ & \\
April, 2014 & F & - & - & $-17.13-256.18$ \\
& M & 14 & $83.50-219.05$ & $5.10-21.10$ \\
May, 2014 & F & 17 & $89.70-112.40$ & $13.90-20.00$ \\
& M & 12 & $80.30-115.80$ & $15.50-17.10$ \\
June, 2014 & F & 13 & $103.70-230.12$ & $15.40-17.60$ \\
& M & 9 & $108.80-240.68$ \\
\hline
\end{tabular}

Table 2. Sex ratio and percentage abundance of Synodontis membranaceus in the Lower Benue River.

\begin{tabular}{|c|c|c|c|c|c|c|c|}
\hline \multirow{2}{*}{ Months } & \multirow{2}{*}{ M } & \multirow{2}{*}{$\mathbf{F}$} & \multirow{2}{*}{ Total } & \multirow{2}{*}{ Sex Ratio (M:F) } & \multicolumn{3}{|c|}{ Percentage (\%) abundance } \\
\hline & & & & & M & $\mathbf{F}$ & Combined \\
\hline July, 2013 & 27 & 17 & 44 & $1.6: 1$ & 13.37 & 8.42 & 21.78 \\
\hline September, 2013 & 12 & 15 & 27 & $1: 1.3$ & 5.94 & 7.43 & 13.37 \\
\hline October, 2013 & 1 & 1 & 2 & $1: 1$ & 0.50 & 0.50 & 1.00 \\
\hline November, 2013 & 2 & 2 & 4 & $1: 1$ & 0.99 & 0.99 & 1.98 \\
\hline January, 2014 & 1 & 0 & 1 & $1: 0$ & 0.5 & - & 0.5 \\
\hline February, 2014 & - & - & - & - & - & - & - \\
\hline March, 2014 & - & - & - & - & - & - & - \\
\hline April, 2014 & 14 & 17 & 31 & $1: 1.2$ & 6.93 & 8.42 & 15.35 \\
\hline May, 2014 & 12 & 13 & 25 & $1: 1.1$ & 5.94 & 6.44 & 12.38 \\
\hline June, 2014 & 9 & 11 & 20 & $1: 1.2$ & 4.46 & 5.45 & 9.90 \\
\hline
\end{tabular}

Table 3. Monthly variation in condition factor, $a, b$ and $r$ values of Synodontis membranaceus from July, 2013 to June, 2014.

\begin{tabular}{|c|c|c|c|c|c|c|}
\hline Month & Sex & No. & K range & $\mathbf{a}$ & b & $\mathbf{r}$ \\
\hline \multirow{3}{*}{ July, 2013} & M & 27 & $1.81-2.82$ & -1.45 & 2.87 & 0.98 \\
\hline & $\mathrm{F}$ & 17 & $2.19-2.65$ & -1.55 & 2.95 & 1.00 \\
\hline & Combined & 44 & $1.81-2.82$ & -1.49 & 2.90 & 0.99 \\
\hline \multirow{4}{*}{ August, 2013} & $\mathrm{M}$ & 22 & $1.88-3.36$ & -1.15 & 2.61 & 0.97 \\
\hline & $\mathrm{F}$ & 25 & $1.83-3.14$ & -1.29 & 2.74 & 0.98 \\
\hline & Combined & 47 & $1.88-3.36$ & -1.27 & 2.72 & 0.98 \\
\hline & M & 12 & $2.12-3.02$ & -0.76 & 2.33 & 0.95 \\
\hline \multirow[t]{2}{*}{ September, 2013} & $\mathrm{~F}$ & 15 & $2.13-2.75$ & 0.67 & 1.27 & 0.69 \\
\hline & Combined & 27 & $2.12-3.02$ & 0.26 & 1.53 & 0.73 \\
\hline \multirow{3}{*}{ October, 2013} & M & 1 & $0.00-2.52$ & - & - & - \\
\hline & $\mathrm{F}$ & 1 & $0.00-2.68$ & - & - & - \\
\hline & Combined & 2 & $0.00-2.68$ & - & - & - \\
\hline \multirow{3}{*}{ November, 2013} & $\mathrm{M}$ & 2 & $2.23-2.32$ & -0.99 & 2.25 & 1.00 \\
\hline & $\mathrm{F}$ & 2 & $2.19-2.23$ & -1.63 & 3.03 & 1.00 \\
\hline & Combined & 4 & $2.19-2.32$ & -1.68 & 2.98 & 1.00 \\
\hline \multirow{3}{*}{ December, 2013} & M & 1 & $0.00-2.42$ & - & - & - \\
\hline & $\mathrm{F}$ & - & - & - & - & - \\
\hline & Combined & 1 & $0.00-2.42$ & - & _ & - \\
\hline \multirow{3}{*}{ January, 2014} & M & 1 & $0.00-1.81$ & - & - & - \\
\hline & $\mathrm{F}$ & 0 & - & - & - & - \\
\hline & Combined & 1 & $0.00-1.81$ & _- & - & _- \\
\hline \multirow{3}{*}{ April, 2014} & M & 14 & $2.20-3.05$ & -0.52 & 2.09 & 0.95 \\
\hline & $\mathrm{F}$ & 17 & $2.32-3.13$ & -1.46 & 2.89 & 0.95 \\
\hline & Combined & 31 & $2.20-3.05$ & -0.58 & 2.15 & 0.95 \\
\hline \multirow{3}{*}{ May, 2014} & M & 12 & $2.03-2.51$ & -0.16 & 1.77 & 0.69 \\
\hline & $\mathrm{F}$ & 13 & $2.03-2.41$ & -063 & 2.16 & 0.89 \\
\hline & Combined & 25 & $2.03-2.51$ & -0.57 & 2.11 & 0.84 \\
\hline \multirow{3}{*}{ June, 2014} & M & 9 & $2.20-2.58$ & -1.26 & 2.72 & 0.99 \\
\hline & $\mathrm{F}$ & 11 & $2.27-2.76$ & -1.24 & 2.72 & 0.96 \\
\hline & Combined & 20 & $2.20-2.76$ & -1.22 & 2.69 & 0.98 \\
\hline
\end{tabular}




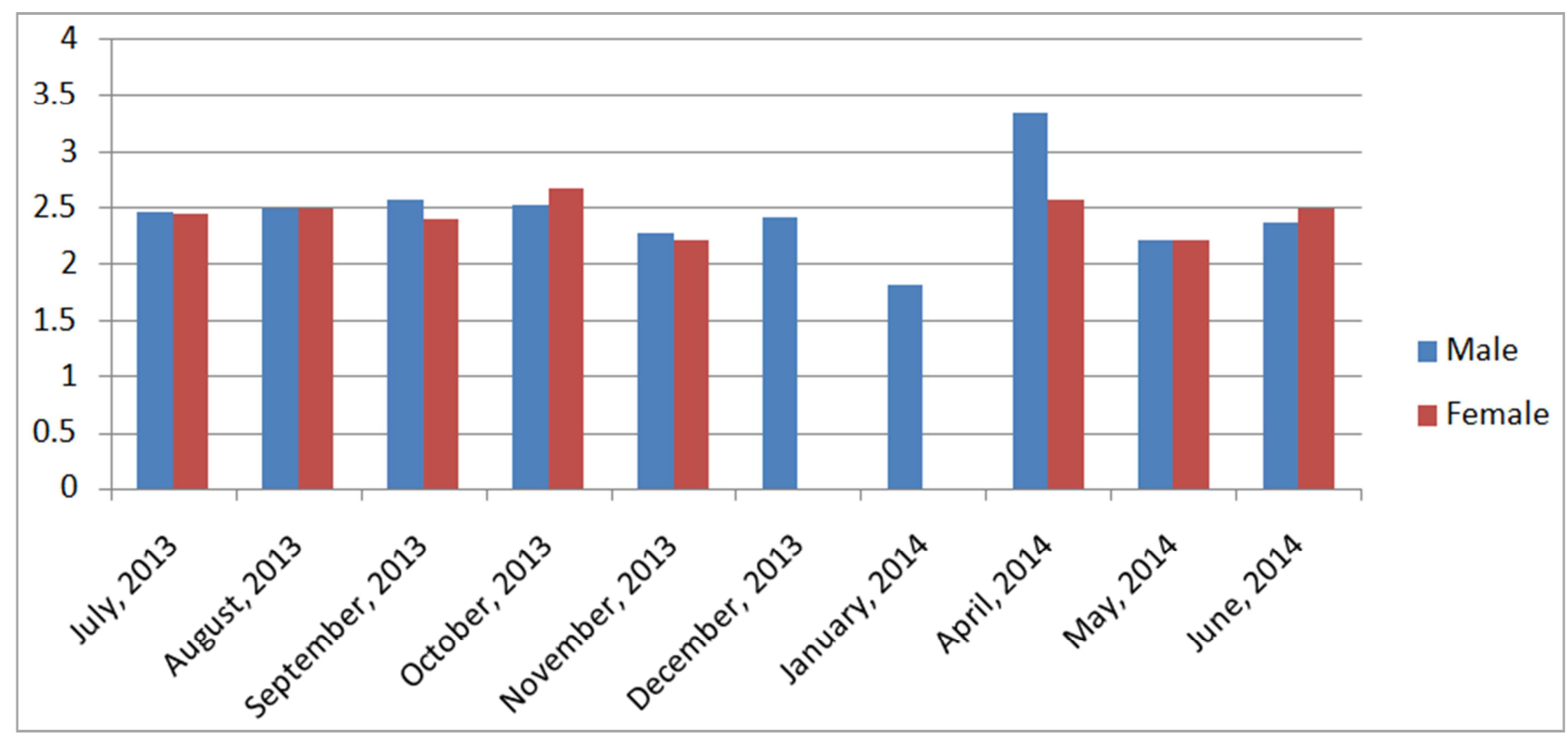

Fig. 1. Mean monthly condition factor of S. membranaceus from lower Benue River.

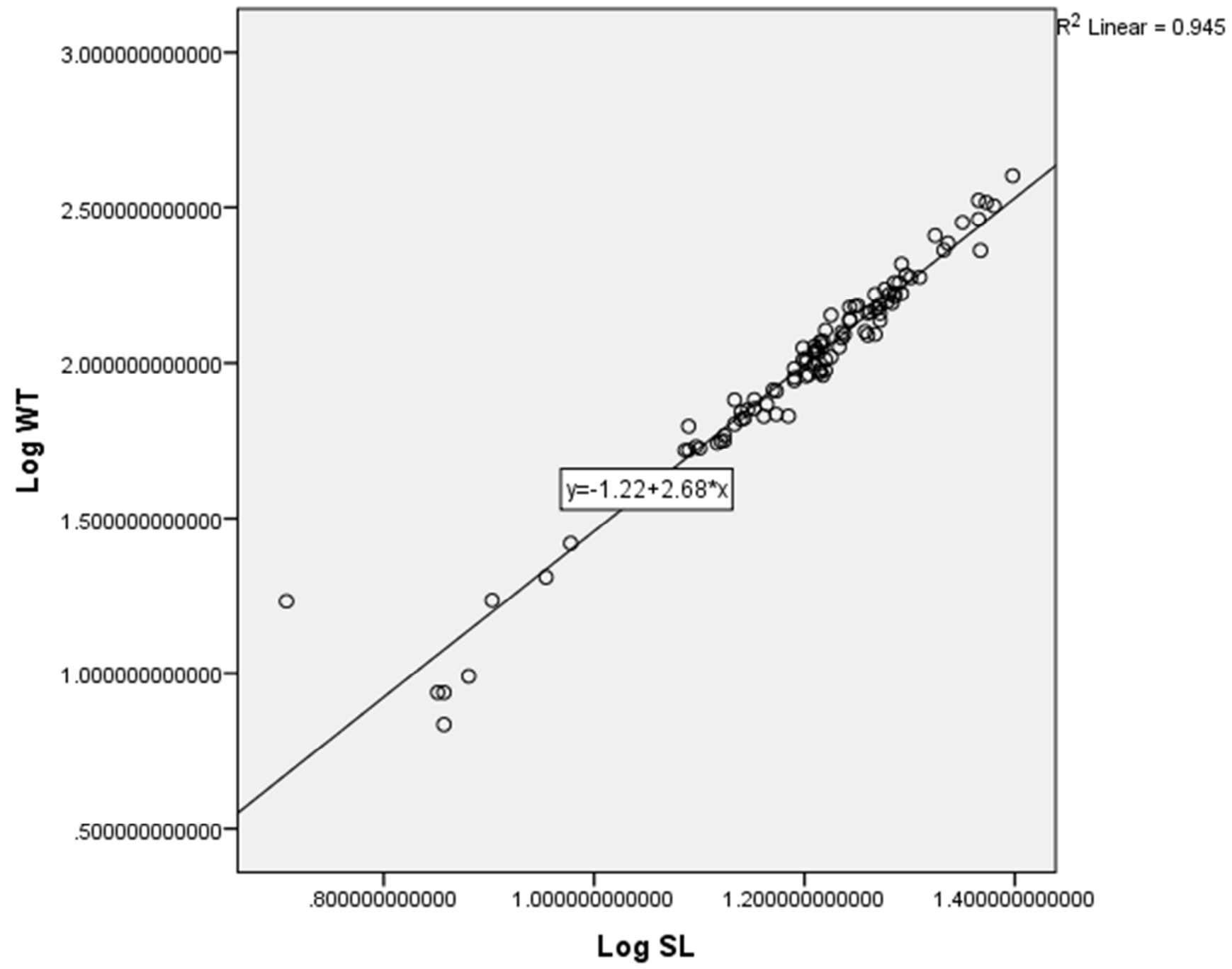

Fig. 2. Length-weight relationship of S. membranaceus (male). 


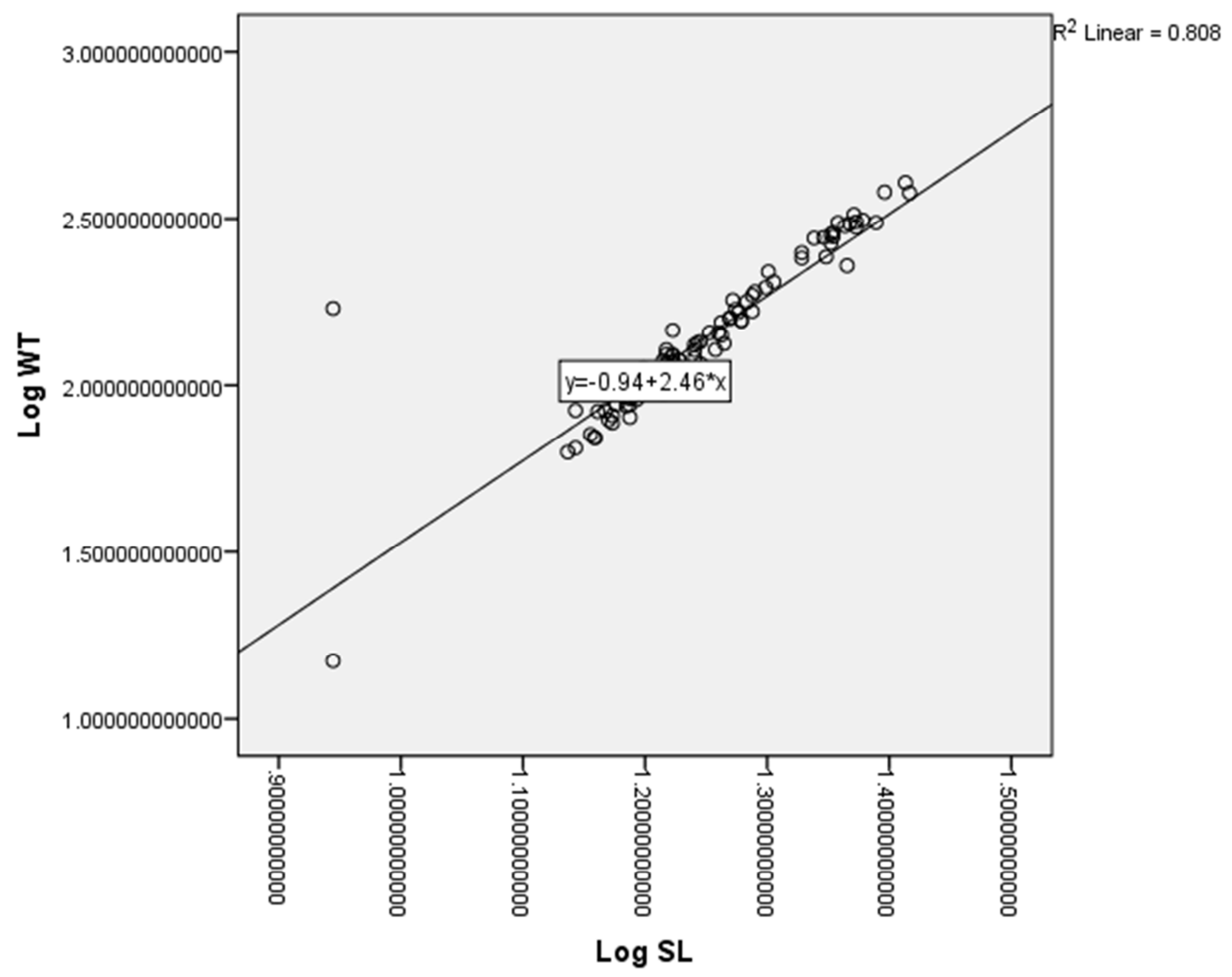

Fig. 3. Length-weight relationship of S. membranaceus (female).

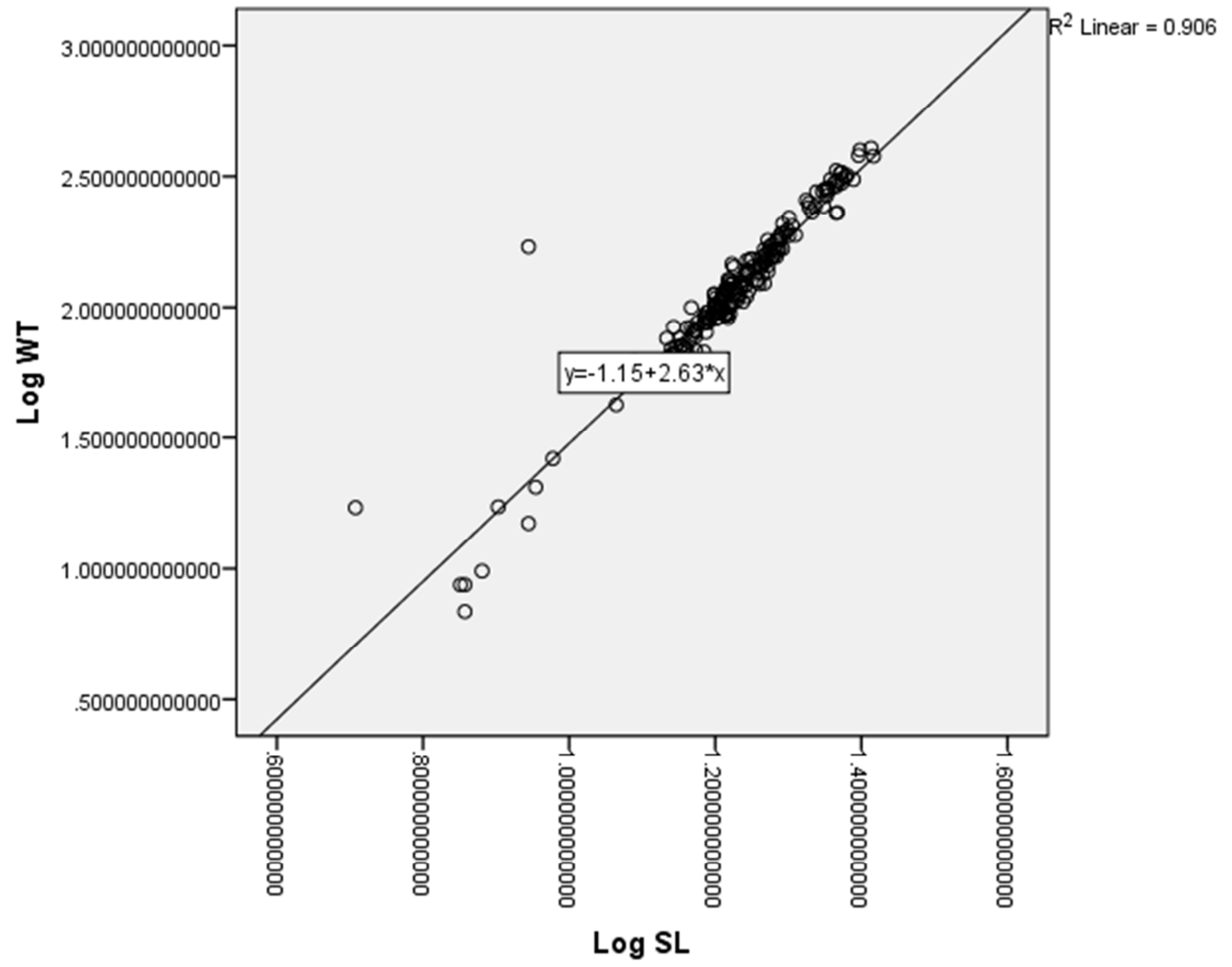

Fig. 4. Length-weight relationship of S. membranaceus (combined). 
Table 4. Stomach content of S. membranaceus in the lower Benue River using frequency of occurrence Method.

\begin{tabular}{|c|c|c|c|c|c|c|c|c|c|c|}
\hline Food item & Jul., 2013 & Aug., 2013 & Sept., 2013 & Oct., 2013 & Nov., 2013 & Dec., 2013 & Jan., 2014 & Apr., 2014 & May, 2014 & Jun., 2014 \\
\hline \multicolumn{11}{|l|}{ PLANT } \\
\hline Plant remains & 94.87 & 68.29 & 70.83 & 50.00 & 50.00 & 100.00 & 100.00 & 67.74 & 48.00 & 80.00 \\
\hline Artificial meal & 79.49 & 31.71 & 37.50 & 50.00 & 25.00 & 100.00 & _- & 9.68 & - & - \\
\hline Millet & 2.56 & - & - & - & - & - & - & & - & - \\
\hline $\begin{array}{l}\text { Maize } \\
\text { ALGAE }\end{array}$ & 5.13 & - & - & - & - & - & - & 3.23 & - & - \\
\hline Diatoms & 87.81 & 80.49 & 87.50 & 100.00 & 100.00 & 100.00 & 100.00 & 87.10 & 80.00 & 65.00 \\
\hline Surface water algae & 15.38 & 70.73 & 70.83 & 50.00 & 75.00 & _ & 100.00 & 70.97 & 92.00 & 100.00 \\
\hline Pigmented flagellates & 10.26 & & 4.17 & _- & _ & _ & _- & _ & & 55.00 \\
\hline Filamentous algae & 35.90 & 7.32 & _- & _- & _- & _- & _- & _ & 76.00 & 55.00 \\
\hline $\begin{array}{l}\text { Blue green algae } \\
\text { INSECTS }\end{array}$ & - & - & - & - & - & - & - & - & 12.00 & 35.00 \\
\hline Insect larvae & 64.10 & 7.76 & 12.50 & 50.00 & 75.00 & & & 35.48 & 4.00 & \\
\hline $\begin{array}{l}\text { Insect parts } \\
\text { MOLLUSCS }\end{array}$ & 71.79 & 68.29 & 91.67 & 50.00 & 75.00 & $\overline{1} 00.00$ & $\overline{1} 00.00$ & 54.85 & 36.00 & $\overline{5} .00$ \\
\hline $\begin{array}{l}\text { Bivalves } \\
\text { CRUSTACEANS }\end{array}$ & 2.56 & 4.88 & 4.17 & - & 25.00 & 100.00 & - & - & - & - \\
\hline Crustacean parts & 51.28 & 53.66 & 58.33 & 50.00 & 25.00 & 100.00 & _ & 35.48 & 32.00 & 55.00 \\
\hline $\begin{array}{l}\text { Crustacean eggs } \\
\text { PROTOZOA }\end{array}$ & - & - & - & - & - & - & - & 9.68 & 24.00 & 5.00 \\
\hline Paramecia & - & - & - & - & - & - & _- & 3.23 & 12.00 & 15.00 \\
\hline $\begin{array}{l}\text { Amoebae } \\
\text { ROTIFERS }\end{array}$ & - & - & - & - & - & - & - & - & - & - \\
\hline $\begin{array}{l}\text { Rotifer parts } \\
\text { WORMS }\end{array}$ & 7.69 & 9.76 & 8.33 & - & - & - & - & 3.23 & 32.00 & 70.00 \\
\hline Round worms & 76.92 & 73.17 & 25.00 & & & & & 16.31 & 8.00 & 10.00 \\
\hline Detritus & 71.79 & 92.68 & 91.67 & 100.00 & 100.00 & 100.00 & 100.00 & 74.19 & 52.00 & 80.00 \\
\hline Sand particles/mud & 89.74 & 70.73 & 45.83 & 100.00 & 75.00 & 100.00 & _ & 54.84 & 8.00 & 15.00 \\
\hline Polythene & & & & & & _ & & 3.23 & & \\
\hline Unidentified items & 82.05 & 85.36 & $\overline{4} 1.67$ & 100.00 & 100.00 & & 100.00 & 74.19 & 92.00 & $\overline{7} 5.00$ \\
\hline
\end{tabular}

Table 5. Stomach content of S. membranaceus in Lower Benue River using Numerical Method.

\begin{tabular}{|c|c|c|c|c|c|c|c|c|c|c|}
\hline Food item & Jul., 2013 & Aug., 2013 & Sept., 2013 & Oct., 2013 & Nov., 2013 & Dec., 2013 & Jan., 2014 & Apr., 2014 & May, 2014 & Jun., 2014 \\
\hline \multicolumn{11}{|c|}{ (1) - } \\
\hline Grains & 0.63 & & & & & & & 0.44 & & \\
\hline Plant remains & $*$ & $\bar{*}$ & $\bar{*}$ & $\bar{*}$ & * & $\bar{*}$ & $\bar{*}$ & $*$ & $\bar{*}$ & $\bar{*}$ \\
\hline $\begin{array}{l}\text { Artificial meal } \\
\text { ALGAE }\end{array}$ & $*$ & $*$ & $*$ & $*$ & $*$ & $*$ & $*$ & $*$ & * & $*$ \\
\hline Diatoms & 27.22 & 21.22 & 31.75 & 25.00 & 18.18 & 40.00 & 22.22 & 27.31 & 16.42 & 11.99 \\
\hline Surface water algae & 4.11 & 16.72 & 22.22 & 12.50 & 12.12 & 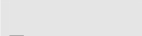 & 22.22 & 24.23 & 28.44 & 37.83 \\
\hline Pigmented flagellates & - & - & _- & _- & _- & - & _ & _- & & 6.74 \\
\hline Filamentous algae & 6.01 & 1.61 & - & - & - & 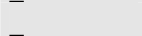 & - & - & 9.28 & 4.49 \\
\hline $\begin{array}{l}\text { Blue green algae } \\
\text { INSECTS }\end{array}$ & - & - & - & - & - & - & - & - & 0.90 & 2.62 \\
\hline Insect larvae & 8.86 & 1.29 & 2.38 & 6.25 & 9.09 & & & 4.85 & 0.30 & \\
\hline $\begin{array}{l}\text { Insect parts } \\
\text { MOLLUSCS }\end{array}$ & 9.81 & 10.29 & 18.25 & 6.25 & 9.09 & $\overline{4} 0.00$ & $\overline{1} 1.11$ & 8.37 & 2.69 & $\overline{0} .37$ \\
\hline $\begin{array}{l}\text { Bivalves } \\
\text { CRUTACEANS }\end{array}$ & 0.32 & 0.64 & 0.79 & _- & 3.03 & 20.00 & - & - & - & - \\
\hline Crustacean parts & $*$ & $*$ & * & $*$ & $*$ & * & $*$ & $*$ & * & * \\
\hline $\begin{array}{l}\text { Crustacean eggs } \\
\text { PROTOZOA }\end{array}$ & $*$ & $*$ & $*$ & $*$ & $*$ & * & $*$ & $*$ & $*$ & * \\
\hline Paramecia & _- & - & - & - & - & - & _- & 0.44 & 0.90 & \\
\hline $\begin{array}{l}\text { Amoebae } \\
\text { ROTIFERS }\end{array}$ & - & - & - & - & - & - & - & - & - & 1.12 \\
\hline $\begin{array}{l}\text { Rotifers parts } \\
\text { WORMS }\end{array}$ & 1.58 & 1.61 & 1.59 & - & - & - & - & 0.44 & 2.40 & 5.24 \\
\hline Round worms & 10.13 & 11.58 & 5.56 & & & & & 2.20 & 0.60 & 0.75 \\
\hline Detritus & $*$ & $*$ & $*$ & $\bar{*}$ & $\bar{*}$ & $\bar{*}$ & $\bar{*}$ & $*$ & $*$ & $*$ \\
\hline Sand particles/mud & $*$ & $*$ & $*$ & $*$ & $*$ & $*$ & $*$ & $*$ & * & $*$ \\
\hline Polythene & _- & - & - & & - & - & & 0.44 & & \\
\hline Unidentified items & 29.43 & 35.05 & 17.46 & 50.00 & 48.48 & & $\overline{4} 4.22$ & 29.96 & 36.23 & 28.46 \\
\hline
\end{tabular}

*Undeterminable using this method. 
Atile John I. et al:: Food and Feeding, Length-Weight and Condition Factor of the Catfish Synodontis membranaceus

(EtieneGeoffroy Saint Hilaire, 1809) (Osteichthyes: Mochokidae) from Lower Benue River, Makurdi, Nigeria

Table 6. Stomach content of S. membranaceus in the Lower Benue River using Point Method.

\begin{tabular}{|c|c|c|c|c|c|c|c|c|c|c|}
\hline Food item & Jul., 2013 & Aug., 2013 & Sept., 2013 & Oct., 2013 & Nov., 2013 & Dec., 2013 & Jan., 2014 & Apr., 2014 & May, 2014 & Jun., 2014 \\
\hline PLANT & & & & & & & & & & \\
\hline Plant remains & 13.61 & 10.33 & 11.70 & 7.50 & 7.50 & 15.00 & 15.00 & 12.66 & 6.90 & 10.89 \\
\hline Artificial meal & 7.33 & 4.50 & 6.89 & 10.00 & 3.75 & 15.00 & - & 1.76 & - & - \\
\hline Millet & 0.25 & - & _ & - & _ & _- & - & - & - & - \\
\hline $\begin{array}{l}\text { Maize } \\
\text { ALGAE }\end{array}$ & 0.35 & - & - & - & - & - & - & 0.32 & - & - \\
\hline Diatoms & 15.30 & 13.64 & 17.34 & 15.00 & 15.00 & 10.00 & 25.00 & 18.11 & 13.81 & 9.90 \\
\hline Pigmented flagellates & 0.96 & _ & 0.42 & - & _ & - & - & _ & _- & 7.18 \\
\hline Filamentous algae & 4.18 & 1.27 & _ & - & - & - & - & - & 13.61 & 9.65 \\
\hline $\begin{array}{l}\text { Blue green algae } \\
\text { INSECTS }\end{array}$ & - & - & - & - & - & - & - & - & 1.18 & 3.22 \\
\hline Insect larvae & 6.29 & 0.99 & 1.67 & 7.50 & 10.00 & - & - & 4.81 & 0.39 & - \\
\hline $\begin{array}{l}\text { Insect parts } \\
\text { MOLLUSCS }\end{array}$ & 7.67 & 7.84 & 13.16 & 7.50 & 10.00 & 10.00 & 10.00 & 6.89 & 4.54 & 0.50 \\
\hline $\begin{array}{l}\text { Bivalves } \\
\text { CRUSTACEANS }\end{array}$ & 0.25 & 0.38 & 0.42 & - & 2.50 & 10.00 & - & - & - & - \\
\hline Crustacean parts & 4.85 & 5.77 & 8.15 & 7.50 & 2.50 & 15.00 & - & 4.49 & 3.35 & 6.19 \\
\hline $\begin{array}{l}\text { Crustacean eggs } \\
\text { PROTOZOA }\end{array}$ & - & - & - & - & - & - & - & 1.12 & 2.37 & 0.50 \\
\hline Paramecia & - & - & - & - & - & - & - & 0.32 & 1.18 & - \\
\hline $\begin{array}{l}\text { Amoebae } \\
\text { ROTIFERS }\end{array}$ & - & - & - & - & - & - & - & 0.36 & 3.16 & 6.93 \\
\hline $\begin{array}{l}\text { Rotifer parts } \\
\text { WORMS }\end{array}$ & 0.69 & 1.02 & 0.84 & - & - & - & - & 0.32 & 3.16 & 6.93 \\
\hline Round worms & 7.20 & 7.94 & 2.51 & - & - & - & & 1.60 & 0.79 & 0.74 \\
\hline Detritus & 6.56 & 16.23 & 17.50 & 17.50 & 17.50 & 15.00 & 25.00 & 17.95 & 9.07 & 12.87 \\
\hline Sand particles/mud & 8.42 & 7.61 & 4.59 & 10.00 & 7.50 & 10.00 & - & 5.45 & 0.79 & 2.97 \\
\hline $\begin{array}{l}\text { Polythene } \\
\text { Unidentified items }\end{array}$ & $\overline{13.89}$ & $\overline{13.30}$ & $\overline{5} .01$ & $\overline{7} .50$ & $\overline{13} .75$ & _- & $\overline{10.00}$ & $\begin{array}{l}0.16 \\
10.90\end{array}$ & $\overline{19.13}$ & $\overline{9} .41$ \\
\hline
\end{tabular}

Table 7. Stomach vacuity index of males and females of S. membranaceus in the Lower Benue River from July, 2013 to June, 2014.

\begin{tabular}{|c|c|c|c|c|c|c|c|}
\hline Month & Total & TSEM & ESM & TSEF & ESF & SVIM (\%) & SVIF (\%) \\
\hline July, 2013 & 44 & 27 & 2 & 17 & 3 & 7.41 & 17.65 \\
\hline August, 2013 & 47 & 22 & 6 & 25 & 0 & 27.27 & 0 \\
\hline September, 2013 & 27 & 12 & 1 & 15 & 2 & 8.33 & 13.33 \\
\hline October, 2013 & 2 & 1 & _ & 1 & _- & 0 & 0 \\
\hline November, 2013 & 4 & 2 & - & 2 & - & 0 & 0 \\
\hline December, 2013 & 1 & 1 & - & _- & - & 0 & _- \\
\hline January, 2014 & 1 & 1 & - & - & - & 0 & - \\
\hline February, 2014 & - & - & - & - & - & - & - \\
\hline March, 2014 & - & 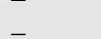 & - & 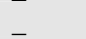 & - & - & - \\
\hline April, 2014 & $\overline{31}$ & $\overline{14}$ & - & $\overline{17}$ & - & $\overline{0}$ & $\overline{0}$ \\
\hline May, 2014 & 25 & 12 & - & 13 & - & 0 & 0 \\
\hline June, 2014 & 20 & 9 & & 11 & & 0 & 0 \\
\hline
\end{tabular}

$\mathrm{TSEM}=$ Total stomach examined in males, ESM=Empty stomach in males, TSEF=Total stomach examined in females, ESF=Empty stomach in females, $\mathrm{SVIM}=$ Stmach vacuity index in males and $\mathrm{SVIF}=$ Stomach vacuity index in females

\section{Discussion}

The length-weight relationships of the males, females and combined sexes of $S$. membranaceus were highly significantly correlated $(\mathrm{P}<0.01)$ with high positive correlation coefficient (r). The values of regression coefficient (b) observed in this study were significantly below 2.99, which indicated that both male and female species of $S$. membranaceus exhibited negative allometric growth pattern. The $\mathrm{b}$ values of $2.2749,2.2915$ and 2.2863 were observed for male, female and combined sexes of $S$. schall respectivelyin River Nile at Gizza, Egypt (Midhat et al., 2012). The observations in this study are also in agreement withfour species of Synodontis in Lower Benue River, (Akombo et al 2011); for S. schall in River Nile at Assiut (Hassan 2007); and S. nigrita in Ouèmè River Benin (Lalèyè et al., 2006).

S. membranaceus had equal number of males and females on the whole during the study period. However, they were varied number of males and females in some months. Equal availability of males and females on the whole and as well as none availability of fish in February and March, 2014 could be attributed to the type of gears used, length of sampling period, time of sampling, seasonal changes, food availability, changes in water quality and the migration of the fish to shoals due to reduction in water level. 
According to Khallaf and Authman (2010), sex ratio in fishes varied from one species to another. Nieto-Navarro et al., (2010) concluded that the differences in observations could be due to seasonal variability of the environment, food availability, and sample size and length interval within different areas or habitat suitability. Akombo et al., (2011) observed that $S$. membranaceus had more females than males with the male to female sex ratio of 1:1.6 and were not found in March, April and October, 2009 while $S$. schall had the sex ratio of 1.1:1.1 and were found throughout the sampling period. Midhat (2012) reported that the number of males of $S$. schall exceeded that of the females with the sex ratio of 1.2:1.

The lower and the higher values of mean condition factor (k) recorded for males of $S$. membranaceus implies that they could survive better even when biotic and abiotic factors are less favourable (Akombo et al., 2011). Abowei (2009) reported the condition factor of Hemisynodontis membranaceus in the freshwater Reaches of Lower Num River, Niger Delta to be 0.83-1.00. Midhat et al., (2012) reported that the males of $S$. schall had better conditions (1.83) than females in River Nile at Gizza. The different values of mean k (1.81-3.35 for males and 2.21-2.68 for females) obtained in this study could be as a result of differences in gonadal maturation, increase or decrease in feeding activities, population changes possibly due to modifications in food resources (Akombo et al., 2011), as well as the general well being of the individual fish species. $S$. membranaceus was in good condition as all the values of $\mathrm{k}$ for the males and females were within the recommended ranged of 2.9-4.8 for fresh water fishes (Bagenal and Tesch, 1978).

The stomach vacuity indices of $S$. membranaceus showed that both the males and the females were not highly selective in their feeding and were flexible in their diets in many months during the sampling period. However, the species sexes tended to select food in few months. This could be attributed to differences in their taste, size of the fish, variation in food availability, changes in weather conditions and habitat preferences by the fish. Akombo (2014) reported the highest vacuity index in $S$. sorex $(83.33 \%)$ and the lowest in $S$. batensoda (0.00) in the lower Benue River, and attributed that species like $S$. sorex, $S$. violaceus, $S$. clarias, $S$. courteti, S. filamentous, S. vemiculatus and $S$. nigrita were not very common in the river because of high selectivity exhibited in their feeding habits and concluded that species like $S$. schall, S. gambiensis and S. membranaceus were more flexible in their diets and were more abundant.

Out of 202 stomachs of $S$. membranaceus examined for the food content analysis, 9 males $(8.91 \%)$ and 5 females $(4.95 \%)$ had empty stomachs while $91.09 \%$ of males and $95.05 \%$ of females contained varied quantities of food items. Results on stomach content as presented in Tables 5, 6 and 7 showed that specimens of $S$. membranaceus examined during the study period were omnivorous. Meye et al., (2008) reported that $S$. ocellifer from River Adofi in Southern Nigeria fed on a wide spectrum of food items ranging from various types of algae, detritus, sand particles and mud indicating that the species exhibited omnivorous feeding.
Akombo et al., (2011) also made similar observations on four species of Synodontis in the Lower Benue River at Makurdi.

This study showed that there was abundance of food for this fish and the fish fed well both in the months of wet and dry seasons. The high diversity of the food composition in the stomachs of $S$. membranaceus indicated a wide adaptability to the food and feeding habit in the Lower Benue River in which they live. This is an important strategy for survival and an advantage over the fishes competing for a specific food item (Lalèyè et al., 2006).

\section{Conclusion}

S. membranaceus in the Lower Benue River was found to be omnivorous, feeding on a wide variety of food items. The fish fed well during the months of wet and dry seasons.

\section{Acknowledgments}

Authors wish to express their profound appreciation to the Department of Biological Sciences, Benue State University; to the laboratory technicians, Mr. Waya, J. I., Mrs Shiriki D., Mrs. Tyona E., Mr. Adanu, P. Mrs Adejoh M., Mr. Girinya, B. for their technical support during the implementation of this study.

\section{References}

[1] Abowei, J. F. N. (2009). Aspects of Hemisynodontis membanaceus (Geoffrey- St, Hilare, 1809) Population Dynamics from the Fresh Water Reaches of Lower Nun River, Niger Delta, Nigeria. Advance Journal of Food and Technology, 1 (1): 27-34.

[2] Adeyemi, S. O. (2011). Study of some physico-chemical parameters and their effect on Potential Fish Yield in Gbadikere Lake. Bassa, Kogi State, Nigeria. Pakistan Journal of Nutrition, 10 (5): 475-479.

[3] Akange, E. T (2011). Feeding Ecology of four Catfishes (Osteichthyes: Siluriformes) in Lower River Benue, Nigeria. M.Sc. Thesis (Unpuplished), Benue State University, Makurdi.

[4] Akombo, P. M., Atile, J. I., Adikwu, I. A. and Araoye, P. A. (2011). Morphometric Measurements and growth patterns of four species of the genus Synodontis (Cuvier, 1816) from Lower Benue River, Makurdi, Nigeria. International Journal of Fisheries and Aquaculture, 3 (15): 263-270.

[5] Akombo, P. M. (2014). Population Dynamics of the Catfish (Synodontis, Cuvier, 1816) in the Lower Benue River, Nigeria. Ph.D Thesis, Benue State University, Makurdi. Unpublished.

[6] Akombo, P. M., Shima, J. N., Adikwu, I. A., and Araoye, P. A. (2010). Intestine to standard length and food habits of Synodontis species from the lower Benue River, Nigeria. Nigerian Journal of Fisheries 7 (1\&2): 8-15.

[7] Bagenal, T. B. (1978). Aspects of fish fecundity, pp 75-95. In: S. D. Gerking (ed) Ecology of Freshwater Fish Production, Blackwell Scientific. Oxford England. 
[8] Bagenal. T. B. and Tesch, F. W. (1978). Methods for assessment of Fish Production I Freshwaters. T. B. Bagenal (9ed.) I. B. P. Handbook No. $33^{\text {rd }}$ edn. Oxford Blackwell Publications, 365 .

[9] Berg. J. (1979). Dicussion of Method Investigating the Food of Fishes with Reference to a Preliminary Study of the prey of Gobiusculus flavescens. Marine Biology, 50: 263-273.

[10] Hassan, A. A. (2007). Studies on Population dynamics of two fresh water fish species: Synodontisschall and Mormyruskannume from Nile at Assiut, Egypt. M.Sc. Thesis. Department of Zoology, Faculty of Science, Assiut university, Assiut, Egypt.

[11] Hynes, H. B. N. (1950). The food of freshwater Stickleback, Gasterosteusaculiatus and Pygosteuspungitius with a review of methods used in studies of the food of fishes. J. Ani. Ecol. 19: $15-36$

[12] Hyslop, E. J. (1980). Stomach Content Analysis-A review of Methods and Their Application. J. Fish. Biol. 17: 411-429.

[13] Khallaf, E. A. and Authman, M. N. (2010). Some biological aspects of the Nile Mormirid fish (Mormyrus kannume, Forsskal, 1775) from Bahr Shebeen Nilotic canal, Egypt. World J. Fish Mar. Sci., 2: 357-375.

[14] Lagler, K. F., Bardach, J. E., Litter, R. R. and Passimo, D. R. M. (1977). Ichthyology. John Wiley and Sons Inc., 506.

[15] Lalèyè, P., Chikou, A., Gnohssou, P., Vandewalle, J. C. P. and Teugels, G. (2006). Studies on the Biology of two species of catfish Synodontis schall and Synodontis nigrita (Ostariphysi: Mochokidae) from the Ouémé River, Bénin. Belg. J. Zool., 136 (2): 193-201.

[16] Meye, J. A., Omoruwou, P. E. and Mayor, E. D. (2008). Food and feeding habits of Synodontis ocellifer (Boulenger, 1900) from River Adofi, Southern Nigeria. Tropical Freshwater Biology, 17 (1): 1-12.

[17] Midhat, A. E. K., Mohammed, M. N., A. and Seham, A. I. (2012). Environmental studies on Synodontisschall (Bloch and
Schneider, 1801), (Pisces: Mochokidae) in The River Nile at Gizza Sector, Egypt: Biological aspects of Population Dynamics. Journal of Fisheries and Aquatic, 7: 104-133.

[18] Nedeco (1959). Studies and Recommendations: Improvement of Niger and Benue Rivers. Amsterdam. North Holland Publishing Company, 19-27.

[19] Nieto-Navarro, J. T., Zetina-Rejon, Arreguin-Sanchez, ArcrosHuitron, N. E. and Petria-Mesina (2010). Length-weight relationships of dermersal fish from the Eastern Coast of California. Journal of fisheries and Aquatic Sciences, 5 (6): 494-502.

[20] Ogbe, F. G., Ataguba, G. A. \& Okosuwe, E. H. (2008). Feeding Habits and growth parameters of Hydrocynusforskaliiand Alestes nurse in River Benue, Makurdi, Nigeria. J. Appl. Biosciences. 11: 576-583.

[21] Ogbe and Fagade 2002 (Please include this reference)

[22] Ogbe, F. G. \& Fagade, S. O. (2002). Distribution, Abundance and Dimensional Features of Clarotes laticeps (Ruppell) and C. macropterus (Daget) in Lower Benue River, Makurdi, Nigeria. Journal of prospects in Sciences. 6: 18-23.

[23] Oronsaye, C. G. \& Nakpodia, F. A. (2005). A Comperative Study of the Food and Feeding Habits of Chrysichthysnigrodigitus and Bryanus nurse in a tropical river. Park J. Sc, ind Res. 48 (2): 118-121.

[24] Owolabi, O. D. (2008). The Dietary Habits of the Upsidedown catfish, Synodontis membranaceus (Osteichthyes: Mochokidae) in Jebba Lake. Rev. Biol. Trop. (International Journal of Tropical Biology), 56 (2): 931-936.

[25] Reid, M. G. and Sydenhan, H. L. (1979). A check-list of Lower Benue River fishes. Icthyo - geographical review of the Benue River, West-Africa. Journal of Natural History, 13: 14-67.

[26] Solomon, S. G., Ataguba, G. A. \& Baiyewunmi, A. S. (2009). Study of Dry Season Zooplankton of lower river Benue at Makurdi, Nigeria. J. Anim. Pl. Sci. 1 (3): 42-50. 